Published in Food and Chemical Toxicology 49 (2011) 750-757

DOI: 10.1016/j.fct.2010.11.037

Copyright Elsevier 2010, all rights reserved

\title{
Cob(I)alamin Reacts with Sucralose to Afford an Alkylcobalamin: Relevance to In Vivo Cobalamin and Sucralose Interaction
}

\author{
Hitesh V. Motwani ${ }^{\mathrm{a}, \mathrm{b}}$, Shiran Qiu ${ }^{\mathrm{a}}$, Bernard T. Golding ${ }^{\mathrm{b}}$, Henrik Kylin ${ }^{\mathrm{c}}$, and Margareta Törnqvist ${ }^{\mathrm{a},{ }^{*}}$ \\ ${ }^{a}$ Department of Materials and Environmental Chemistry, Stockholm University, Stockholm 106 91, Sweden \\ ${ }^{b}$ School of Chemistry, Newcastle University, United Kingdom \\ ${ }^{c}$ Norwegian Institute for Air Research, Polar Environmental Centre, Norway \\ * Corresponding author. Address: Department of Materials and Environmental Chemistry, Environmental Chemistry \\ Unit, Stockholm University, Stockholm 106 91, Sweden. Email: margareta.tornqvist@mmk.su.se; Tel: +46-8-163769; \\ Fax: +46-8-163979
}

\begin{abstract}
Vitamin $\mathrm{B}_{12}$, chemically known as cobalamin, is a complex organometallic cofactor associated with several cobalamin-dependent enzymes. Its reduced form, $\operatorname{cob}(\mathrm{I})$ alamin, is one of the most powerful nucleophiles known. Following reduction of hydroxocobalamin $(\mathrm{OH}-\mathrm{Cbl})$ by sodium borohydride, cob(I)alamin was shown to react readily with sucralose (1',6'-dichloro-1',6'-dideoxy- $\beta$-Dfructofuranosyl-4-chloro-4-deoxy- $\alpha$-D-galactopyranoside; a synthetic sweetener) in an aqueous system to form an alkylcobalamin (Suc-Cbl). This occurred by replacement of one of the three chlorides on sucralose with a cobalamin moiety. The efficiency of trapping sucralose in presence of excess $\mathrm{Cbl}(\mathrm{I})$ was estimated to be $>90 \%$. Furthermore, in an in vitro study using human liver S9 with NADPH regeneration, in presence of $\mathrm{OH}-\mathrm{Cbl}$ and sucralose, Suc-Cbl was shown to be formed. The Suc-Cbl was characterized primarily by LC-ESI ${ }^{+}$-MS/MS. Considering human consumption of sucralose from food and beverages, such reaction between the sweetener and reduced vitamin $B_{12}$ could occur in vivo.
\end{abstract}

\section{Keywords}

Hydroxocobalamin, sucralose, sodium borohydride, NADPH, alkylcobalamin, LC-MS/MS 


\section{Introduction}

Cobalamins (Fig. 1) consist of a central cobalt atom that is coordinated by four coplanar nitrogen ligands in a reduced tetrapyrrole structure representing the corrin ring. In alkylcobalamins (R-Cbls) an alkyl group is bound to the cobalt in the upper axial position, and the lower axial position is occupied by a nitrogen of the dimethylbenzimidazole nucleotide substituent (Hodgkin et al., 1956). Cob(III)alamin species, organo- or alkyl-cobalamins, can be reduced in hepatic cells by NADPH to cob(II)alamin $\{\mathrm{Cbl}(\mathrm{II})\}$ and cob(I)alamin $\{\mathrm{Cbl}(\mathrm{I})\}$ (Banerjee et al., 1990; Leclerc et al., 1998; Olteanu et al., 2001; Watanabe et al., 1992; Watson et al., 2004). Cbl(I) has the ability to react $10^{5}$ times faster than standard nucleophiles (Haglund et al., 2003), and is often denoted as a "supernucleophile" (Schrauzer et al., 1969). The supernucleophilicity of Cbl(I) has been harnessed by a number of cobalamin-dependent enzymes (Banerjee, 1999; Banerjee et al., 2003; Golding et al., 1997; Matthews et al., 2003). For instance, in methionine synthase Cbl(I) serves to transfer a methyl group from $\mathrm{N}^{5}$-methyltetrahydrofolate to homocysteine, yielding methionine and tetrahydrofolate. Another example is human adenosyltransferase, which is an enzyme involved in the biosynthesis of adenosylcobalamin.

Vitamin $\mathrm{B}_{12}$ \{e.g. hydroxocobalamin (OH-Cbl)\}, following reduction to $\mathrm{Cbl}(\mathrm{I})$, is known to react with alkyl halides (Anderson et al., 1992; Schrauzer et al., 1969) and epoxides (Alsberg et al., 2001; Dixon et al., 1983; Fred et al., 2004; Haglund et al., 2006; Haglund et al., 2003; Motwani et al., 2009) to give alkyl- and 2-hydroxyalkyl-cobalamins, respectively, usually by a $\mathrm{S}_{\mathrm{N}} 2$-type mechanism. This could be referred in vivo as scavenging of the alkylating substances by Cbl(I). It has also been shown that Cbl(II) could react with alkyl halides, by a radical mechanism, to give RCbls (Blaser et al., 1980; Watson et al., 2004). The R-Cbls so formed may undergo conversion, e.g. mediated by NADPH, to a reduced cobalamin, which in the absence of reduction reactivation could oxidize to OH-Cbl (Scheme 1).

Herein, we have investigated organic and bio-organic reactions between reduced vitamin $\mathrm{B}_{12}$ and sucralose. The synthetic sweetener sucralose (1',6'-dichloro-1',6'-dideoxy- $\beta$-D-fructofuranosyl4-chloro-4-deoxy- $\alpha$-D-galactopyranoside, also known as trichlorogalactosucrose; for structure see Scheme 2), was first reported in 1975 (Fairclough et al., 1975). The compound is now, because of favorable technical and sensory properties, used in food and beverages as a low calorie sweetener. Sucralose has been allocated an acceptable daily intake of $15 \mathrm{mg} / \mathrm{kg}$ bw (body weight) by the Joint FAO/WHO Expert Group on Food Additives (JECFA, 1991). Because of the three tightly bound chlorine atoms, sucralose is exceptionally stable over a wide temperature and $\mathrm{pH}$ range.

Sucralose has been discussed as a possible human health hazard, mostly in public media because of its chlorinated structure (Abou-Donia et al., 2008; Bigal et al., 2006; Peterson, 2007; Rodero et al., 2009). In a subchronic toxicity test, with $2.8-6.4 \mathrm{~g} / \mathrm{kg}$ bw per day of sucralose administered to rats in the diet, the rats showed several ill-effects. However, it was suggested that most of the effects were related to physiologic changes and palatability of the food, rather than direct toxicity (Goldsmith, 2000). No significant toxicological concern of sucralose have been implied in humans (Brusick et al., 2009; Grice et al., 2000; Grotz et al., 2009; Hagiwara, 1999; Roberts et al., 2000). This could be due to the hydrophilicity ( $\log$ Kow $=-0.5$ ) (Jenner et al., 1989), and relatively low absorption (ca. $14.5 \%$ ) and low metabolism $(<2 \%)$ of the compound in mammals. Conjugation with glutathione has been shown to occur with one of the hydrolyzed metabolites, 1',6'-dichloro-1',6', dideoxy-D-fructose (1,6-DCF), of sucralose (Grice et al., 2000; 
Hughes et al., 1989; Roberts et al., 2000). The other, 4-chloro-4-deoxy-D-galactose (4-CG), excretes in the urine essentially unchanged (Scheme 2).

The present study shows that $\mathrm{Cbl}(\mathrm{I})$ generated in an aqueous solution from $\mathrm{OH}-\mathrm{Cbl}$ can react with sucralose and form an alkylcobalamin (Suc-Cbl). For identification of the product, Suc-Cbl was synthesized as a reference compound. R-Cbls having molecular masses $>1500$ and being polar complexes with good ionization properties were analyzed by LC-ESI-MS/MS. Considering the possible toxicological significance of this reaction in humans, an in vitro ab initio study was performed with presence of $\mathrm{OH}-\mathrm{Cbl}$ and sucralose in human liver S9 containing microsomal and cytosolic fractions with NADPH regeneration. This was to study whether the reaction between reduced vitamin $\mathrm{B}_{12}$ and sucralose could occur in physiological conditions.

\section{Materials and Methods}

Note: All reactions concerning cobalamin were carried out in the dark or when needed under a dim red light.

\subsection{Materials}

Hydroxocobalamin hydrochloride, cyanocobalamin, adenosylcobalamin, cobalt(II) nitrate and $\beta$-nicotinamide adenine dinucleotide phosphate sodium salt (NADP) were obtained from Sigma (St. Louis, MO). Sucralose ( $\geq 98 \%$ ), glucose-6-phosphate, magnesium sulfate, potassium chloride, sodium borohydride, 3-bromo-1-propanol and trifluoroacetic acid were obtained from SigmaAldrich Sweden AB (Stockholm, Sweden). Splenda ${ }^{\circledR}$ was obtained from McNeil Nutritionals LLC (Fort Washington, PA). Ingredients stated in Splenda ${ }^{\circledR}$ were sucralose, dextrose and maltodextrin. Human liver S9 fraction (30 mg/mL proteins) was purchased from Moltox Inc. (Boone, NC) and stored frozen below $-70{ }^{\circ} \mathrm{C}$ until further use. All solvents were of HPLC grade.

\subsection{Synthesis of Suc-Cbl}

Cyanocobalamin (100 mg, $0.07 \mathrm{mmol}$ ) and a catalytic quantity of cobalt(II) nitrate (2 mg, $0.01 \mathrm{mmol})$ in water-ethanol $(8: 2 \mathrm{v} / \mathrm{v}, 10 \mathrm{~mL})$ were deoxygenated at room temperature by bubbling a slow stream of nitrogen for $25 \mathrm{~min}$, followed by addition of sodium borohydride (20 mg, 0.55 $\mathrm{mmol}$ ) in water $(1 \mathrm{~mL})$. After $5 \mathrm{~min}$ sucralose $(200 \mathrm{mg}, 0.5 \mathrm{mmol})$ in water $(1 \mathrm{~mL})$ was added and stirring under nitrogen was continued. The reaction was completed after $2 \mathrm{~h}$ as monitored with TLC. Finally, the reaction was quenched by addition of acetone $(0.1 \mathrm{~mL})$. The aqueous solution of Suc-Cbl was transferred to a separating funnel and extracted with 1-propanol in ethyl acetate (1:3 $\mathrm{v} / \mathrm{v}, 8 \times 5 \mathrm{~mL})$. The organic phase was washed with water $(2 \times 1 \mathrm{~mL})$, and diluted with ethyl acetate $(8 \times$ the original volume of the organic phase). The alkylcobalamin was re-extracted into water $(5 \times$ $5 \mathrm{~mL})$ and the aqueous extract was washed with ethyl acetate $(4 \times 5 \mathrm{~mL})$. The aqueous layer was dried by rotary evaporation and the residual liquid was freeze-dried. The product was reprecipitated from water upon addition of acetone to afford Suc-Cbl (77 mg, 65\%). ${ }^{1} \mathrm{H}$ NMR [500 MHz, $\mathrm{D}_{2} \mathrm{O}$ (for numbering scheme see Fig. 1)]: 5.96 (C10-H), 6.14 (B4-H), 6.18 (R1-H), 6.94 (B2-H), 7.10 (B7-H), and other resonances. ESI ${ }^{+}-\mathrm{MS}: \mathrm{m} / \mathrm{z} 1692[\mathrm{M}]^{+}, 846.5[\mathrm{M}+2 \mathrm{H}]^{2+}, 829[\mathrm{M}-\mathrm{Cl}+2 \mathrm{H}]^{2+}, 811.5[\mathrm{M}-$ $2 \mathrm{Cl}+2 \mathrm{H}]^{2+}, 665.5\left[\mathrm{M}-\mathrm{C}_{12} \mathrm{H}_{19} \mathrm{C}_{12} \mathrm{O}_{8}+2 \mathrm{H}^{2+}\right.$. $\mathrm{R}_{\mathrm{f}} 0.61$. See sections 2.7, 2.8 and 2.9 for detection by TLC, HPLC-UV and LC-MS/MS, respectively.

\subsection{Trapping conditions with $\mathrm{Cbl}(\mathrm{I})$}


The conditions applied were similar to those used for formation of hydroxyalkylcobalamins from oxiranes (Haglund et al., 2006). Briefly, OH-Cbl (5 mM in water, $200 \mu \mathrm{L}$ ) and cobalt(II) nitrate (25 mM in water, $20 \mu \mathrm{L}$ ) were added to a septum sealed amber vial. The solution was degassed with argon for $10 \mathrm{~min}$. Aqueous sodium borohydride $(150 \mathrm{mM}, 50 \mu \mathrm{L})$ was added and the mixture was agitated by argon bubbling. After about 2 min Cbl(I) formation was judged to be complete. 3-Bromo-1-propanol $(0.1 \mathrm{mM}$ in methanol, $200 \mu \mathrm{L})$ was mixed with sucralose $(0.3 \mathrm{mM}$ in water, $200 \mu \mathrm{L}$ ) and $300 \mu \mathrm{L}$ from this mixture was added to Cbl(I) solution. After 30 min under inert atmosphere, air was passed into the reaction mixture for $5 \mathrm{~min}$ to oxidize any remaining reduced cobalamins and the reaction mixture was then analyzed by HPLC-UV and LC-MS/MS.

\subsection{Evaluation of Cbl(I) method for quantitative analysis of Suc-Cbl formation}

$\mathrm{Cbl}(\mathrm{I})$ was generated as described above under trapping conditions. Prior to this, solutions of sucralose $(200 \mu \mathrm{L})$ were prepared in duplicates of 8 different concentrations (0 to $100 \mu \mathrm{M})$ in water. 3-Bromo-1-propanol ( $20 \mu \mathrm{M}$ in methanol, $200 \mu \mathrm{L})$ was added as an internal standard to each sucralose solution. A sample (300 $\mu \mathrm{L})$ from this mixture was added to Cbl(I) solution. After $30 \mathrm{~min}$ under inert atmosphere, air was passed into the reaction mixture for 5 min to oxidize any remaining reduced cobalamins. The analytes were frozen until analysis by LC-MS/MS. A calibration plot was obtained by plotting the area ratios of the Suc-Cbl and 3-hydroxypropylcobalamin (IS-Cbl), formed from sucralose and internal standard respectively, against the concentrations of sucralose.

\subsection{Determination of sucralose in Splenda ${ }^{\circledR}$}

The method applied was similar to that in 2.4 above. The difference was that concentrations of Splenda ${ }^{\circledR}$ (25, 50 and $\left.100 \mathrm{mg}\right)$, each in $100 \mathrm{~mL}$ water, were used instead of sucralose solution. The concentration of sucralose in Splenda ${ }^{\circledR}$ solution was determined from the calibration plot.

\subsection{In vitro reactions under physiological conditions}

To human liver S9 (150 $\mu \mathrm{L}, 4.5 \mathrm{mg}$ proteins) was added $\mathrm{OH}-\mathrm{Cbl}(1 \mathrm{mM}, 20 \mu \mathrm{L}), \mathrm{KCl}(0.15$ $\mathrm{M}, 300 \mu \mathrm{L}), \mathrm{MgSO}_{4}(80 \mathrm{mM}, 150 \mu \mathrm{L})$, glucose-6-phosphate $(50 \mathrm{mM}, 150 \mu \mathrm{L})$ and NADP $(40 \mathrm{mM}$, $150 \mu \mathrm{L})$, and the volume was made up to $1.5 \mathrm{~mL}$ with phosphate buffer $(\mathrm{pH} \mathrm{7.4)}$. The mixture was incubated at $37^{\circ} \mathrm{C}$ for $5 \mathrm{~min}$, prior to addition of sucralose $(20 \mathrm{mM}, 20 \mu \mathrm{L})$. Samples $(250 \mu \mathrm{L})$ were collected from the S9 system between 0 and $80 \mathrm{~min}$ and mixed with ice cold methanol $(250 \mu \mathrm{L})$. The mixture was mixed, centrifuged (3000 rpm, $10 \mathrm{~min}$ ) and the cobalamins were extracted from the supernatant by phenol - dichloromethane (DCM) $(1: 1 \mathrm{w} / \mathrm{v}, 400 \mu \mathrm{L} \times 3)$ and washed with water $(200 \mu \mathrm{L} \times 2)$. Excessive DCM $(10 \mathrm{~mL})$ was added and the cobalamins re-extracted into water $(400$ $\mu \mathrm{L} \times 3)$. The aqueous phase, containing cobalamins, was washed with DCM $(200 \mu \mathrm{L} \times 2)$, and frozen until LC-MS/MS analysis. In control studies, incubations were performed in the absence of sucralose, and in absence of NADP.

The S9 was post mitochondrial supernatant from five human liver samples pooled together. Presence of adventitious agents, promutagen activation and cytochrome P450 (3.34 nmoles/mL) content were provided by the supplier (Moltox). Epoxide hydrolase activity was assessed by hydrolysis of an oxirane (1,2-epoxy-3-butene) as in our earlier work (Motwani et al., 2009).

\subsection{TLC}

TLC analysis was done on silica gel plates (Merck 5554) with 2,2,2-trifluoroethanol- 
dichloromethane-conc. ammonia (5:2:0.08 v/v/v) as eluent. Hydroxo-, cyano-, and adenosylcobalamin were used as references: $\mathrm{R}_{\mathrm{f}} 0.32,0.46$ and 0.62 , respectively.

\subsection{HPLC-UV}

A Shimadzu LC-10 AD system with an ACE 5 C18, $250 \times 4.6 \mathrm{~mm}$ column (from Scantec Lab AB, Sweden) coupled to UV detector (LKB, Bromma, Sweden) set at $267 \mathrm{~nm}$ was used. Two mobile phase systems were used to obtain a linear gradient from $10 \%$ to $40 \%$ acetonitrile in 15 min and then $40 \%$ to $70 \%$ acetonitrile in 5 min, with trifluoroacetic acid (TFA) concentration $0.1 \%$ throughout. Flow rate of $1 \mathrm{~mL} / \mathrm{min}$ was applied with an injection volume of $20 \mu \mathrm{L}$.

\subsection{LC-MS/MS}

A Shimadzu Prominence LC-system coupled to an API 3200 Q trap triple quadrupole mass spectrometer (Applied Biosystems) via an ACE 5 C18, $150 \times 1 \mathrm{~mm}$ analytical column with a $10 \times 1$ mm pre-column (both from Scantec Lab AB, Sweden) was used. The R-Cbls were eluted with gradient conditions using two mobile phase systems referred to as solution A (5\% acetonitrile in water, $0.1 \%$ TFA) and solution B (70\% acetonitrile in water, 0.1\% TFA). A linear gradient was used from $5 \%$ to $20 \%$ solution B in 5 min, which was increased to $60 \%$ in 15 min, followed by $60 \%$ to $100 \%$ in $1 \mathrm{~min}$. Analyte solution $(5 \mu \mathrm{L})$ was injected with a flow rate of $0.1 \mathrm{~mL} / \mathrm{min}$.

An electrospray ionization source operated in the positive ion mode $\left(\mathrm{ESI}^{+}\right)$was used. Acquisition and data processing were done with the Analyst software version 1.5 (Applied Biosystems). Parameters optimised for the ESI were: Ion source temperature $100{ }^{\circ} \mathrm{C}$, collision energy $30 \mathrm{eV}$, declustering potential $25 \mathrm{~V}$, entrance potential $7.5 \mathrm{~V}$, collision cell exit potential 25 $\mathrm{V}$, ion spray voltage $5500 \mathrm{~V}$, curtain gas 20, collision gas 5 , and ion source gas 15 (latter three are arbitrary units from the Analyst software). The mass spectrometer was operated on full scan (FS) and MS/MS modes, which were product ion scan (PIS) and multiple reaction monitoring (MRM). MRM precursor ion to product ion transitions used for quantification of OH-Cbl, Suc-Cbl and ISCbl were $m / z$ 674.2, 846.5 and 695.2, respectively, to 665.5.

\section{Results and Discussion}

\subsection{Formation of an alkylcobalamin from sucralose}

Test of reactivity of Cbl(I) towards sucralose was carried out in an aqueous solution with ca. $10 \times$ excess of Cbl(I) (Scheme 3, Route A). A previously developed method in our earlier work for trapping electrophilic substances by Cbl(I) was employed (Haglund et al., 2006; Motwani et al., 2009). To obtain the Cbl(I)-species, OH-Cbl was reduced by sodium borohydride with cobalt(II) nitrate as reduction catalyst under an inert atmosphere using argon. Cbl(I) generation could be observed by color change of red to brown and finally to blackish green. With addition of sucralose a product assumed to be Suc-Cbl, observed as one peak in LC-MS and HPLC-UV chromatogram, was readily formed. A reference amount of the product was synthesized according to the same route and characterized by LC-MS, NMR, UV-Vis and TLC. The reference was verified to be Suc-Cbl and shown identical to the product formed in the trapping solution.

The reaction of $\mathrm{Cbl}(\mathrm{I})$ with sucralose is proposed to occur via an $\mathrm{S}_{\mathrm{N}} 2$-type mechanism at the least hindered chloromethyl group forming Suc-Cbl. The bulky Cbl(I) is expected to be non reactive 
towards the other two sterically hindered chlorinated carbons on sucralose (Schrauzer et al., 1969). This is supported by the observation of one product in the chromatograms (see below).

In the synthesis of reference Suc-Cbl a modified solvent system of 1-propanol and ethyl acetate $(1: 3 \mathrm{v} / \mathrm{v})$ was employed for isolation of Suc-Cbl from water-ethanol solution. This avoided the use of hazardous phenol used in the standard method (Anderson et al., 1992; McCormick et al., 1971).

\subsection{Characterization}

The reference compound (Suc-Cbl) was characterized with LC-MS/MS with electrospray operated in positive ion mode. The structural information of Suc-Cbl was revealed from the molecular mass ion and fragmentation patterns. In the Suc-Cbl full scan mass spectrum both singly $\left([\mathrm{M}]^{+}, m / z\right.$ 1692) and doubly $\left([\mathrm{M}+2 \mathrm{H}]^{2+}, m / z\right.$ 846.5; base peak) protonated molecular ions were observed. Also seen were the doubly protonated dechlorinated fragment ions corresponding to [M $\mathrm{Cl}+2 \mathrm{H}]^{2+}(\mathrm{m} / \mathrm{z} 829)$ and $[\mathrm{M}-2 \mathrm{Cl}+2 \mathrm{H}]^{2+}(\mathrm{m} / \mathrm{z}$ 811.5). In the LC-MS/MS PIS (Fig. 2), the major fragment from $\mathrm{m} / \mathrm{z} 846.5$ was $\mathrm{m} / \mathrm{z} 665.5\left([\mathrm{Cbl}+2 \mathrm{H}]^{2+}\right.$ ), which is a doubly protonated fragment with loss of the alkyl moiety (sucralose) from the cobalt of the cobalamin. This was accompanied by other specific cobalamin fragments $\mathrm{m} / \mathrm{z} 359$ and 972.5 , for which we propose the structures inserted in Fig. 2 (Alsberg et al., 2001). The fragmentation pattern was used for quantification of Suc-Cbl formation by the Cbl(I) trapping method and detection of Suc-Cbl generated in human liver fractions (see sections 3.3 and 3.4).

In addition the synthesized Suc-Cbl was validated by ${ }^{1} \mathrm{H}$ NMR and absorption spectroscopy. In the former, signals assigned in the region $\delta 5-8$ were those on the aromatic portion of 5,6dimethylbenzimidazole (B2-H, B4-H and B7-H), R1-H on the ribose and $\mathrm{C} 10-\mathrm{H}$ on the corrin. These assignments were sufficient to estimate the purity of the compound to be more than $80 \%$ (Anderson et al., 1992; Dixon et al., 1983). The UV-Vis absorption spectrum of Suc-Cbl in methanol showed a characteristic "atypical spectrum" where the $\gamma$-bands (between $350-380 \mathrm{~nm}$ ) are apparently less well defined (Fig. $3 \mathrm{~A}$ ), as in R-Cbls, indicating formation of a Co-C $\sigma$ bond. Absorptions at 350 and $390 \mathrm{~nm}$, which are typical for cyanocobalamin (starting material in synthesis of Suc-Cbl) and Cbl(I), respectively, were not observed, signifying complete alkylation of $\mathrm{Cbl}(\mathrm{I})$. Upon irradiation of the Suc-Cbl in methanol with visible light under aerobic conditions we obtained aquocobalamin $\left(\mathrm{H}_{2} \mathrm{O}-\mathrm{Cbl}\right)$ (Fig. $3 \mathrm{~B}$ ) with its prominent band at $360 \mathrm{~nm}$ implying homolytic cleavage of the Co-C $\sigma$ bond (Firth et al., 1967; Perry et al., 2003; Schrauzer et al., 1970). Such type of cleavage mechanism by homolysis is similar to that of the Co-C bond on adenosylcobalamin-dependent isomerases generating a protein bound free radical, 5'deoxyadenosyl, that catalyze a 1,2 interchange between a variable substituent and a hydrogen atom on vicinal carbons (Banerjee, 1999; Banerjee et al., 2003; Golding et al., 1997). The means of cellular homolytic activation, however, are still enigmatic.

\subsection{Quantification}

For quantification, Suc-Cbl was analyzed using the HPLC-UV and MRM mode on the LCMS/MS. 3-Bromo-1-propanol, which forms 3-hydroxypropylcobalamin (IS-Cbl) with Cbl(I), was used as an internal standard (IS) in the quantitative analysis. The HPLC-UV chromatogram showing Suc-Cbl and IS-Cbl in a sample, with the synthesized Suc-Cbl as a reference, is shown in Fig. 4. For the MRM transitions, the doubly charged molecular ion $\left([\mathrm{M}+2 \mathrm{H}]^{2+}, \mathrm{m} / \mathrm{z}\right.$ 846.5) was selected as 
precursor ion and the doubly charged fragment ion $\left([\mathrm{Cbl}+2 \mathrm{H}]^{2+}, \mathrm{m} / \mathrm{z} 665.5\right)$ was selected as product ion. The MRM chromatogram is exemplified in Fig. 5, which also shows IS-Cbl derived from 3-bromo-1-propanol. The MRM transition used for IS-Cbl was $\mathrm{m} / \mathrm{z} 695.2$ to 665.5. The mobile phase consisted of acetonitrile/water with $0.1 \%$ TFA. For ion spray MS detection of R-Cbls, pH adjustment of the eluent by TFA gave better reproducibility and chromatographic separation compared to formic acid or $\mathrm{HCl}$ or without any pH adjustment (Alsberg et al., 2001).

To study the reproducibility and yield of the trapping of sucralose by Cbl(I) in an aqueous solution, a linearity test was performed. Different concentrations of sucralose (0 to $100 \mu \mathrm{M}$ ) were added, together with a fixed concentration of the internal standard, to a Cbl(I) solution and the formed R-Cbls (Suc-Cbl and IS-Cbl) were quantified by LC-MS/MS using the MRM mode. A linear relationship was found on the calibration curve between peak area ratios of Suc-Cbl to IS-Cbl and sucralose concentrations for duplicate samples with good reproducibility (Fig. 6). The trapping efficiency of sucralose by $\mathrm{Cbl}(\mathrm{I})$ was estimated to be $>90 \%$, by comparing with known concentrations of synthesized Suc-Cbl. Along with validation for formation of Suc-Cbl, the linearity also indicates that the trapping method by Cbl(I) could be developed for analysis of sucralose. With a lowest acceptable signal to noise ratio of 7 , the LOQ for sucralose by the trapping method was determined to be $40 \mu \mathrm{g} \mathrm{L^{-1 }}$.

We tested the $\mathrm{Cbl}(\mathrm{I})$ method for the analysis of sucralose in a commercial product, Splenda ${ }^{\circledR}$, and found the concentration of sucralose $(3.6 \% \pm 0.1 \mathrm{w} / \mathrm{w}, \mathrm{n}=3)$ to be similar to that determined by others (Hanko et al., 2004; Qiu et al., 2007) (data not shown on validation of Cbl(I) as an analytical tool to measure sucralose from complex mixtures).

\subsection{Reactions in human S9}

We considered the possibility that $\mathrm{Cbl}(\mathrm{I})$ generated from reduction of cobalamin species could scavenge the sucralose in vivo (Scheme 1), resembling the reactions with alkyl halides and oxiranes in aqueous solutions (Alsberg et al., 2001; Anderson et al., 1992; Dixon et al., 1983; Fred et al., 2004; Haglund et al., 2006; Haglund et al., 2003; Motwani et al., 2009; Schrauzer et al., 1969). To examine this we used an in vitro system made up of human liver S9 containing OH-Cbl. It is known that cobalamin-dependent methionine synthase in mammals, including humans, contains the cofactor methylcobalamin (Met-Cbl), whereby Cbl(I) is formed as a key intermediate in the cycle of transmethylation reaction resulting in formation of methionine (Banerjee et al., 2003; Banerjee et al., 1990). Reductive reactivation is essential for constant generation of Cbl(I) as oxidative inactivation to $\mathrm{Cbl}(\mathrm{II})$ and $\mathrm{OH}-\mathrm{Cbl}$ could occur that may hinder this reaction (Banerjee et al., 2003; Drummond et al., 1993). NADPH and NADH, amongst several other reductants, can support methionine synthase activity. However, this occurs with different reduction efficiencies. Olteanu and Banerjee suggested the preferred agent to be NADPH, by single electron donation, with an apparent $K_{m}$ of $2.6 \mu \mathrm{M}$ and formation of an air stable semiquinone (Olteanu et al., 2001). Thus, we used NADPH regeneration in our in vitro study as catalyst for reduction of OH-Cbl (Scheme 3, Route B). From pilot studies we determined that reduction of $\mathrm{OH}-\mathrm{Cbl}$ by NADPH generated in human S9 takes about 30 min (Watson et al., 2004).

Under physiological conditions, at $37^{\circ} \mathrm{C}$ and $\mathrm{pH}$ 7.4, sucralose (concentration $20 \times \mathrm{OH}-\mathrm{Cbl}$ ) was added to the human S9 system and aliquots were collected at 0, 15, 30, 40, 60 and $80 \mathrm{~min}$. The cobalamins were isolated from the aliquots by extraction with phenol - dichloromethane (Mahoney et al., 1971) and analyzed by LC-MS/MS. Suc-Cbl was thereby detected at $40 \mathrm{~min}$ and $60 \mathrm{~min}$ in 
the S9. At 40 min, concentration of Suc-Cbl could be quantified by comparing to synthesized Suc$\mathrm{Cbl}$, and was estimated to be ca. $8 \%$ of the initial $\mathrm{OH}-\mathrm{Cbl}$ concentration. Suc-Cbl was not detected at the earlier time points and later at $80 \mathrm{~min}$. The pattern of formation and then disappearance of Suc-Cbl over time was also observed in two other similar incubations from different human S9 batches. This suggested that the Suc-Cbl formed at a time could get reduced further, possibly also by NADPH (Scheme 1). In control studies without sucralose or without NADP (i.e. no NADPH generation), Suc-Cbl was not observed.

The ESI ${ }^{+}$-MS spectrum of Suc-Cbl (Fig. 7) detected in the human S9 system was dominated by the doubly protonated molecular ion $(\mathrm{m} / \mathrm{z}$ 846.5). Also seen were the mono- and di-dechlorinated doubly protonated fragments of Suc-Cbl corresponding to $\mathrm{m} / \mathrm{z} 829.1$ and 811.6. In PIS of Suc-Cbl ( $\mathrm{m} / \mathrm{z}$ 846.5), as in Fig. 2, $\mathrm{m} / \mathrm{z} 665.5$ was the major fragment, along with $\mathrm{m} / \mathrm{z} 359$ and 972.5 .

As sucralose could metabolize by hydrolysis under the in vitro conditions, we also searched for cobalamin adducts generated from the potential reaction of Cbl(I) with 1,6-DCF (cf. Scheme 2). This was performed by LC-MS using extracted ion chromatography for both possible singly and doubly charged ions. However, no R-Cbl from 1,6-DCF was detected in the S9 system. This was not surprising, as the metabolism of sucralose is known to be slow and we had a total incubation time of only 80 minutes (Grice et al., 2000; Roberts et al., 2000).

In the control study without NADP, OH-Cbl levels remained relatively consistent indicating no reduction. $\mathrm{OH}-\mathrm{Cbl}$ levels, though monitored at all times, are not reported as the principal objective of this study was to demonstrate the formation of Suc-Cbl. Moreover, levels of OH-Cbl after reduction could be affected not only by sucralose, but also proteins and other substances such as glucose-6-phosphate (G6P) in the S9. In a preliminary study with Cbl(I) generation in an aqueous solution, an R-Cbl from G6P was formed, whereby the cobalamin moiety replaced the phosphate group (Motwani H. V. et al., unpublished work).

\section{Conclusions and implications}

We have shown that $\mathrm{Cbl}(\mathrm{I})$ reacts with sucralose and forms an alkylcobalamin (Suc-Cbl). This reaction was initially performed in an aqueous system using sodium borohydride as the reducing agent for $\mathrm{OH}-\mathrm{Cbl}$. The efficiency for the trapping of sucralose by $\mathrm{Cbl}(\mathrm{I})$ in the aqueous system was estimated to be $>90 \%$ and the formation of Suc-Cbl was validated by a linearity test. The alkylcobalamin was thoroughly analyzed by LC-MS/MS.

It was also demonstrated that Suc-Cbl can be formed in human liver S9, in an in vitro study. $\mathrm{OH}-\mathrm{Cbl}$, following reduction by NADPH, reacted with sucralose in the S9 system and afforded Suc-Cbl. Cobalamin as a cofactor is present in mammalian liver, including humans, which are reduced by NADPH to $\mathrm{Cbl}(\mathrm{I})$. The reduced vitamin $\mathrm{B}_{12}$ derivatives react with electrophilic substances and form cobalamin adducts. From our work we have shown that Suc-Cbl could be one such adduct. There is a possibility that such reactions, in vivo, may affect the cobalamin levels, analogous to that discussed with exposure to epoxides and nitrous oxide (Deacon et al., 1978; Watson et al., 2004).

With regard to environmental aspects, the demonstrated reaction between $\mathrm{Cbl}(\mathrm{I})$ and sucralose could be useful in the perspective of dehalogenation of the sweetener (Loos et al., 2009; Mead et al., 2009). The formation of Suc-Cbl is a dehalogenation process, mediated by reduced 
vitamin $\mathrm{B}_{12}$, with formation of a Co-C bond. Further reduction of Suc-Cbl by dithiothreitol or titanium(III) citrate could occur resulting in replacement of the cobalamin moiety by a hydrogen atom. In similar contexts metallocoenzymes have previously been used for reductive dechlorination of compounds such as DDT, chloroethylene, hexachlorohexane and hexachlorobenzene in waste treatment and bio-remediation of contaminated water and soil (Assaf-Anid et al., 1992; McCauley et al., 2003; Pratt et al., 2006; Rodriguez-Garrido et al., 2004).

\section{Acknowledgements}

Financial support from the Swedish Research Council Formas, the Swedish Cancer and Allergy Foundation, and the Norwegian Research Council is acknowledged. Prof. Yoshio Hisaeda is thanked for generously providing access to Bruker Avance 500 spectrometer and Hitachi U-3300 spectrophotometer.

\section{References}

Abou-Donia,M.B., El-Masry,E.M., Abdel-Rahman,A.A., McLendon,R.E., and Schiffman,S.S., 2008. Splenda alters gut microflora and increases intestinal p-glycoprotein and cytochrome p-450 in male rats. J Toxicol Environ Health A 71, 1415-1429.

Alsberg,T., Minten,J., Haglund,J., and Tornqvist,M., 2001. Determination of hydroxyalkyl derivatives of cobalamin (vitamin B12) using reversed phase high performance liquid chromatography with electrospray tandem mass spectrometry and ultraviolet diode array detection. Rapid Commun Mass Spectrom 15, 2438-2445.

Anderson,R.J., Dixon,R.M., and Golding,B.T., 1992. Alkylcobalamins: formation by enantioselective alkylation of cob(I)alamin, proton NMR spectra, and conformational analysis of the alkyl group. J. Organomet. Chem. 437, 227-237.

Assaf-Anid,N., Nies,L., and Vogel,T.M., 1992. Reductive dechlorination of a polychlorinated biphenyl congener and hexachlorobenzene by vitamin B12. Appl. Environ. Microbiol. 58, 1057-1060.

Banerjee,R., 1999. Chemistry and Biochemistry of $\mathrm{B}_{12}$.

Banerjee,R. and Ragsdale,S.W., 2003. The many faces of vitamin $\mathrm{B}_{12}$ : catalysis by cobalamindependent enzymes. Annu. Rev. Biochem. 72, 209-247.

Banerjee,R.V. and Matthews,R.G., 1990. Cobalamin-dependent methionine synthase. FASEB J 4, 1450-1459.

Bigal,M.E. and Krymchantowski,A., V, 2006. Migraine triggered by sucralose--a case report. Headache 46, 515-517.

Blaser,H.U. and Halpern,J., 1980. Reactions of vitamin B12r with organic halides. J. Am. Chem. Soc. 102, 1684-1689. 
Brusick,D., Borzelleca,J.F., Gallo,M., Williams,G., Kille,J., Wallace Hayes,A., Xavier PiSunyer,F., Williams,C., and Burks,W., 2009. Expert Panel report on a study of Splenda in male rats. Regul. Toxicol. Pharmacol. 55, 6-12.

Deacon,R., Lumb,M., Perry,J., Chanarin,I., Minty,B., Halsey,M.J., and Nunn,J.F., 11-11-1978. Selective inactivation of vitamin B12 in rats by nitrous oxide. Lancet 2, 1023-1024.

Dixon,R.M., Golding,B.T., Howarth,O.W., and Murphy,J.L., 1983. Synthesis and characterization by proton NMR spectroscopy of diastereoisomeric hydroxy- and dihydroxyalkylcobalamins. J. Chem. Soc. ,Chem. Commun. 243-245.

Drummond,J.T., Huang,S., Blumenthal,R.M., and Matthews,R.G., 9-14-1993. Assignment of enzymatic function to specific protein regions of cobalamin-dependent methionine synthase from Escherichia coli. Biochemistry 32, 9290-9295.

Fairclough,P.H., Hough,L., and Richardson,A.C., 1975. Sucrochemistry. XVI. Derivatives of beta D-fructofuranosyl alpha -D-galactopyranoside. Carbohydr. Res. 40, 285-298.

Firth,R.A., Hill,H.A.O., Pratt,J.M., Williams,R.J.P., and Jackson,W.R., 1967. Circular Dichroism and Absorption Spectra of Some Vitamin B12 Derivatives. Biochemistry 6, 2178-+.

Fred,C., Haglund,J., Alsberg,T., Rydberg,P., Minten,J., and Tornqvist,M., 2004. Characterization of alkyl-cobalamins formed on trapping of epoxide metabolites of 1,3-butadiene. J. Sep. Sci. 27, 607-612.

Golding,B.T. and Buckel,W., 1997. Comprehensive Biological Catalysis. 239-259.

Goldsmith,L.A., 2000. Acute and subchronic toxicity of sucralose. Food Chem. Toxicol. 38, S53S69.

Grice,H.C. and Goldsmith,L.A., 2000. Sucralose - an overview of the toxicity data. Food Chem. Toxicol. 38, S1-S6.

Grotz,V.L. and Munro,I.C., 2009. An overview of the safety of sucralose. Regul. Toxicol. Pharmacol. 55, 1-5.

Hagiwara,A., 1999. Metabolism and safety assessment of sucralose. Foods Food Ingredients J. Jpn. 182, 42-48.

Haglund,J., Silvari,V., Esmans,E., and Tornqvist,M., 6-30-2006. Cobalamin as an analytical tool for analysis of oxirane metabolites of 1,3-butadiene: development and validation of the method. J Chromatogr A 1119, 246-250.

Haglund,J., Magnusson,A.L., Ehrenberg,L., and Toernqvist,M., 2003. Introduction of cob(I)alamin as an analytical tool: application to reaction-kinetic studies of oxiranes. Toxicol. Environ. Chem. 85, 81-94.

Hanko,V.P. and Rohrer,J.S., 7-14-2004. Determination of sucralose in Splenda and a sugar-free beverage using high-performance anion-exchange chromatography with pulsed amperometric detection. J. Agric. Food Chem. 52, 4375-4379. 
Hodgkin,D.C., Kamper,J., Mackay,M., Pickworth,J., Trueblood,K.N., and White,J.G., 1956. Structure of vitamin B12. Nature 178, 64-66.

Hughes,H.M., Powell,G.M., Snodin,D.J., Daniel,J.W., Crawford,A., Sanders,J.K., and Curtis,C.G., 4-15-1989. Glutathione-dependent dechlorination of 1,6-dichloro-1,6-dideoxyfructose. Biochem. J. 259, 537-543.

JECFA, Evaluation of certain food additives and contaminants. Thirty-seventh report of the Joint FAO/WHO Expert Group on Food Additives. WHO Technical Report Series 806. WHO. Geneva. 1991.

Jenner,M.R. and Smithson,A., 1989. Physicochemical Properties of the Sweetener Sucralose. J. Food Sci. 54, 1646-1649.

Leclerc,D., Wilson,A., Dumas,R., Gafuik,C., Song,D., Watkins,D., Heng,H.H., Rommens,J.M., Scherer,S.W., Rosenblatt,D.S., and Gravel,R.A., 3-17-1998. Cloning and mapping of a cDNA for methionine synthase reductase, a flavoprotein defective in patients with homocystinuria. Proc Natl Acad Sci U S A 95, 3059-3064.

Loos,R., Gawlik,B.M., Boettcher,K., Locoro,G., Contini,S., and Bidoglio,G., 2009. Sucralose screening in European surface waters using a solid-phase extraction-liquid chromatographytriple quadrupole mass spectrometry method. J. Chromatogr. A 1216, 1126-1131.

Mahoney,M.J. and Rosenberg,L.E., 1971. Synthesis of cobalamin coenzymes by human cells in tissue culture. J. Lab. Clin. Med. 78, 302-308.

Matthews,R.G., Smith,A.E., Zhou,Z.S., Taurog,R.E., Bandarian,V., Evans,J.C., and Ludwig,M., 2003. Cobalamin-dependent and cobalamin-independent methionine synthases: Are there two solutions to the same chemical problem? Helv. Chim. Acta 86, 3939-3954.

McCauley,K.M., Wilson,S.R., and van der Donk,W.A., 4-16-2003. Characterization of chlorovinylcobalamin, a putative intermediate in reductive degradation of chlorinated ethylenes. J. Am. Chem. Soc. 125, 4410-4411.

McCormick,D.B., Wright,L.D., Colowick,S.P., and Kaplan,N.O., 1971. Methods in Enzymology, 18, Vitamins and Coenzymes, Part C. 34-44.

Mead,R.N., Morgan,J.B., Avery,G.B., Kieber,R.J., Kirk,A.M., Skrabal,S.A., and Willey,J.D., 2009. Occurrence of the artificial sweetener sucralose in coastal and marine waters of the United States. Mar. Chem. 116, 13-17.

Motwani,H.V., Fred,C., Haglund,J., Golding,B.T., and Tornqvist,M., 2009. Cob(I)alamin for trapping butadiene epoxides in metabolism with rat S9 and for determining associated kinetic parameters. Chem. Res. Toxicol. 22, 1509-1516.

Olteanu,H. and Banerjee,R., 9-21-2001. Human methionine synthase reductase, a soluble P-450 reductase-like dual flavoprotein, is sufficient for NADPH-dependent methionine synthase activation. Journal of Biological Chemistry 276, 35558-35563. 
Perry,C.B., Fernandes,M.A., Brown,K.L., Zou,X.A., Valente,E.J., and Marques,H.M., 2003. Probing the nature of the Co-III ion in cobalamins - Spectroscopic and structural investigations of the reactions of aquacobalamin (vitamin B-12a) with ambident nucleophiles. European Journal of Inorganic Chemistry 2095-2107.

Peterson,G., 2007. Sukralos och sötningsmedel. Sveriges konsumenter i samverkan. http://www.konsumentsamverkan.se/11verk/kampanj/lightprd/GPetterssonsuppstas.pdf.

Pratt,D.A. and van der Donk,W.A., 2-7-2006. On the role of alkylcobalamins in the vitamin B12catalyzed reductive dehalogenation of perchloroethylene and trichloroethylene. Chem. Commun. (Camb. ) 558-560.

Qiu,W., Wang,Z., Nie,W., Guo,Y., and Huang,L., 2007. GC-MS determination of sucralose in splenda. Chromatographia 66, 935-939.

Roberts,A., Renwick,A.G., Sims,J., and Snodin,D.J., 2000. Sucralose metabolism and pharmacokinetics in man. Food Chem Toxicol 38 Suppl 2, S31-S41.

Rodero,A.B., Rodero,L.D., and Azoubel,R., 2009. Toxicity of Sucralose in Humans: A Review. International Journal of Morphology 27, 239-244.

Rodriguez-Garrido,B., Arbestain,M.C., Monterroso,M.C., and Macias,F., 10-1-2004. Reductive dechlorination of alpha-, beta-, delta-, and gamma-hexachlorocyclohexane isomers by hydroxocobalamin in the presence of either dithiothreitol or titanium(III) citrate as reducing agents. Environ. Sci. Technol. 38, 5046-5052.

Schrauzer,G.N. and Deutsch,E., 6-4-1969. Reactions of cobalt(I) supernucleophiles. The alkylation of vitamin B12s cobaloximes(I), and related compounds. J. Am. Chem. Soc. 91, 3341-3350.

Schrauzer,G.N., Lee,L.P., and Sibert,J.W., 5-20-1970. Alkylcobalamins and alkylcobaloximes. Electronic structure, spectra, and mechanism of photodealkylation. J Am Chem Soc 92, 2997-3005.

Watanabe,F., Nakano,Y., Saido,H., Tamura,Y., and Yamanaka,H., 2-26-1992. NADPH-cytochrome c (P-450) reductase has the activity of NADPH-linked aquacobalamin reductase in rat liver microsomes. Biochim Biophys Acta 1119, 175-177.

Watson,W.P., Munter,T., and Golding,B.T., 2004. A new role for glutathione: protection of vitamin $\mathrm{B}_{12}$ from depletion by xenobiotics. Chem. Res. Toxicol. 17, 1562-1567.

\section{Figure captions}


Fig. 1. Cobalamin structures.

Fig. 2. $\mathrm{ESI}^{+}$-MS/MS PIS spectrum of Suc-Cbl ( $\mathrm{m} / \mathrm{z}$ 846.5) with proposed structures of the fragments.

Fig. 3. UV-Vis absorption spectra of A: Suc-Cbl in methanol, and B: after irradiation of A with visible light under aerobic conditions.

Fig. 4. HPLC-UV chromatogram showing peaks for A: OH-Cbl, Suc-Cbl and IS-Cbl in order from left to right (insert shows expanded region from 15 to $19 \mathrm{~min}$ ), and B: synthesized Suc-Cbl.

Fig. 5. $\mathrm{LC}-E S I^{+}$-MS/MS MRM spectrum showing Suc-Cbl (left peak) and IS-Cbl (right peak).

Fig. 6. Linearity test for formation of Suc-Cbl. Duplicate samples were taken for each sucralose concentration $(n=8 \times 2)$. $r^{2}=0.998$.

Fig. 7. $\mathrm{ESI}^{+}$-MS spectrum of Suc-Cbl. Insert shows $\mathrm{m} / \mathrm{z}$ for the singly charged molecular ion.
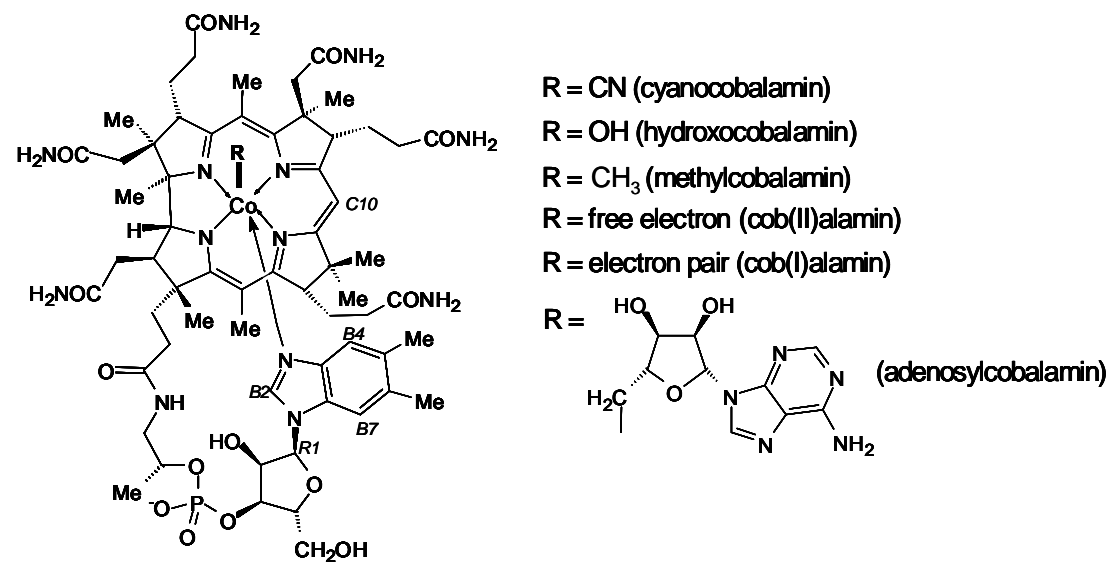

Fig. 1. Cobalamin structures. 


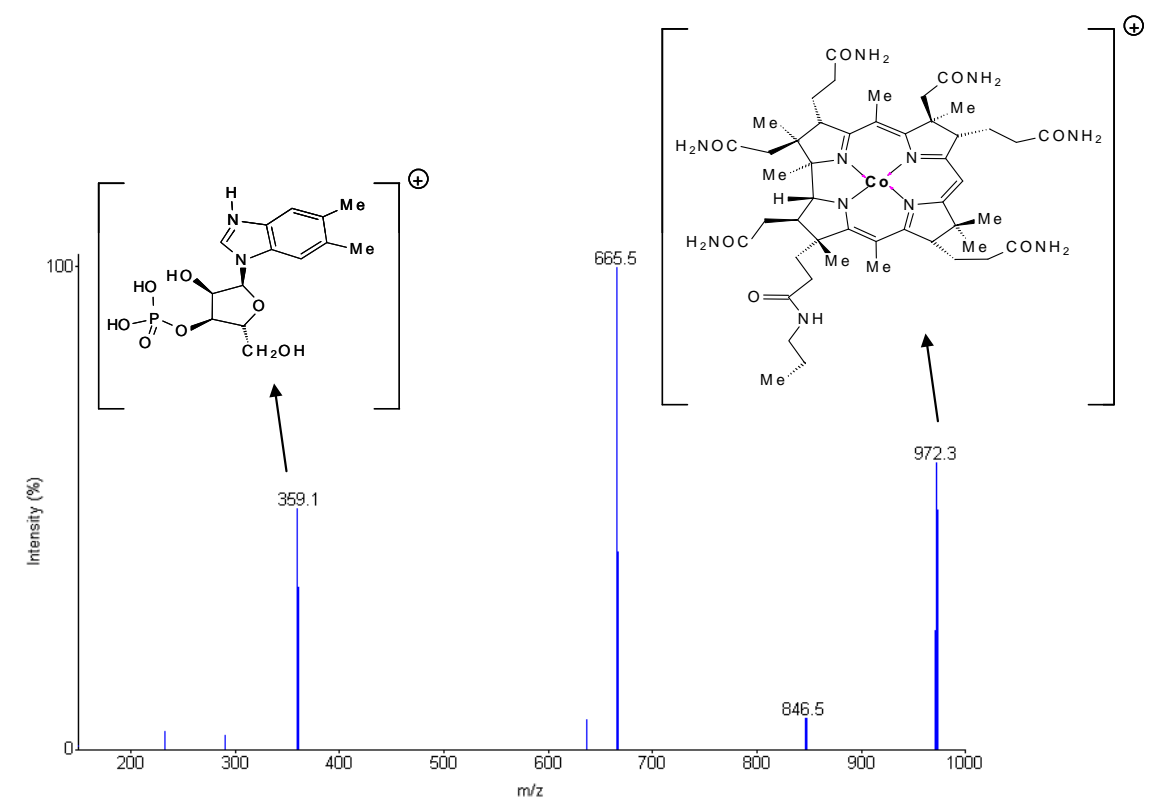

Fig. 2. $\mathrm{ESI}^{+}$-MS/MS PIS spectrum of Suc-Cbl ( $\mathrm{m} / \mathrm{z}$ 846.5) with proposed structures of the fragments.

Fig. 3. UV-Vis absorption spectra of A: Suc-Cbl in methanol, and B: after irradiation of $\mathbf{A}$ with visible light under aerobic conditions. 


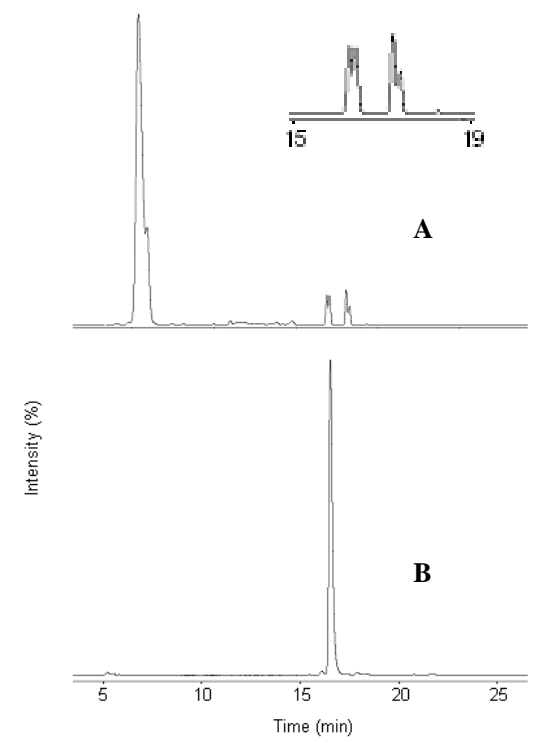

Fig. 4. HPLC-UV chromatogram showing peaks for A: OH-Cbl, Suc-Cbl and IS-Cbl in order from left to right (insert shows expanded region from 15 to $19 \mathrm{~min}$ ), and B: synthesized Suc-Cbl.

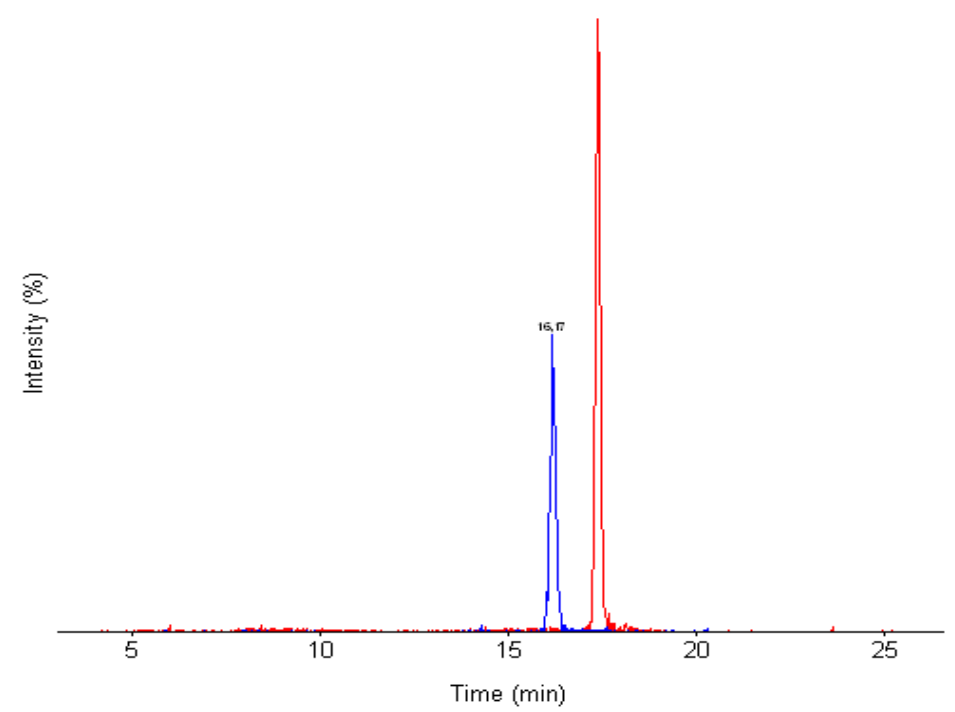

Fig. 5. LC-ESI ${ }^{+}$-MS/MS MRM spectrum showing Suc-Cbl (left peak) and IS-Cbl (right peak). 


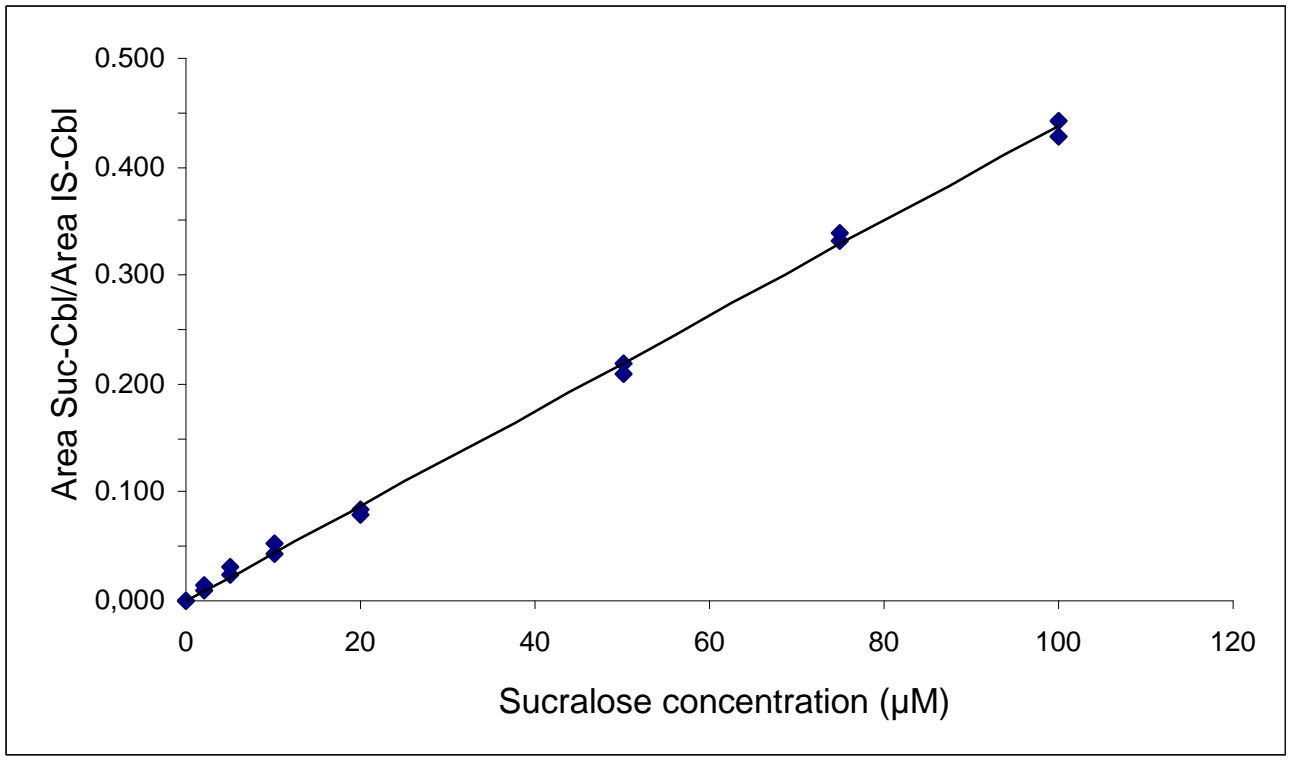

Fig. 6. Linearity test for formation of Suc-Cbl. Duplicate samples were taken for each sucralose concentration $(n=8 \times 2) . r^{2}=0.998$.

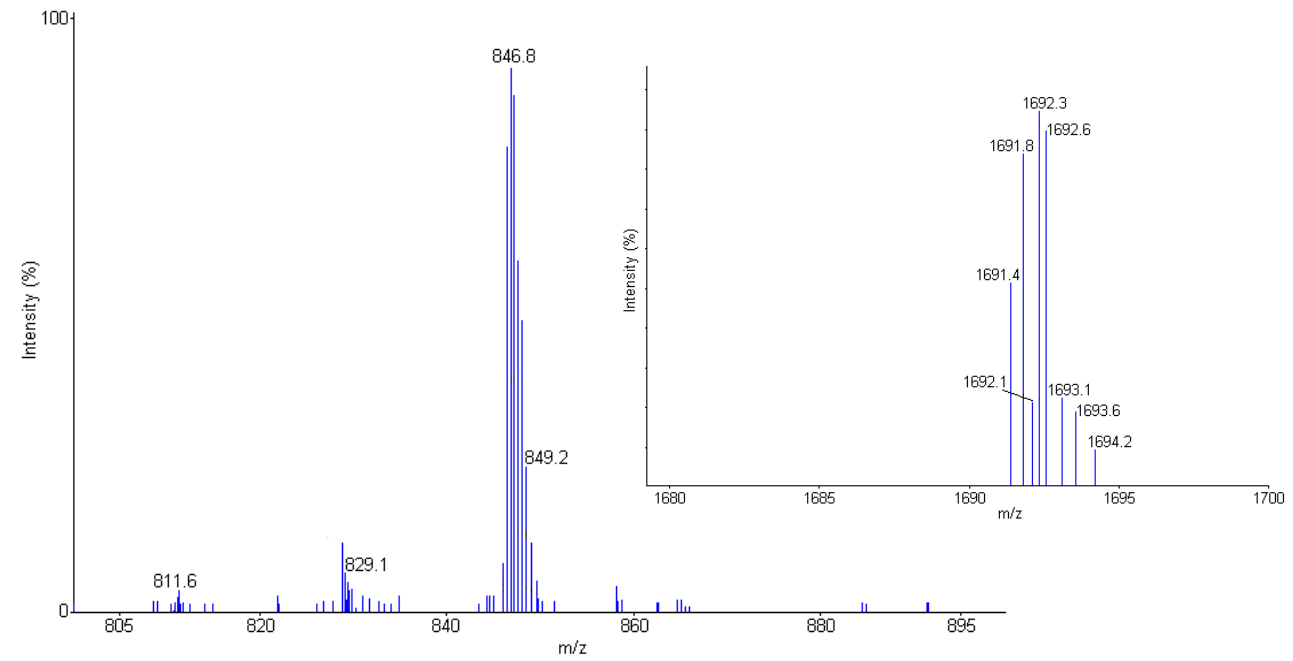

Fig. 7. $\mathrm{ESI}^{+}$-MS spectrum of Suc-Cbl. Insert shows $\mathrm{m} / \mathrm{z}$ for the singly charged molecular ion.

\section{Scheme captions}

Scheme 1. In vitro formation of alkylcobalamin.

Scheme 2. Sucralose and its hydrolyzed products.

Scheme 3. Formation of Suc-Cbl. 
Scheme 1. In vitro formation of alkylcobalamin.

Scheme 2. Sucralose and its hydrolyzed products.

Scheme 3. Formation of Suc-Cbl. 